\title{
Análisis De La Internacionalización De Una Universidad Pública Estatal A Través De La Movilidad Estudiantil
}

\author{
Divad Dinorah García García \\ Dra. Fabiola de Jesús Mapén Franco \\ Dr. Jorge Alberto Rosas Castro
}

Universidad Juárez Autónoma de Tabasco/Villahermosa, Tabasco, México.

doi: 10.19044/esj.2017.v13n10p328 URL:http://dx.doi.org/10.19044/esj.2017.v13n10p328

\begin{abstract}
Internationalization in higher education is a complex process derived from the new social, cultural, and economic dynamics that was generated in national and international context. It was conceived as a strategy to guarantee society with better job opportunities. It starts with the social relevance of the curriculum and the development of institutional programs that favors it. For Higher Education Institutions (IES) to enter into this dynamic process, Knight (1994) describes the integration of the international and intercultural dimensions in the teaching, research, and service functions of the institution. In this sense, the objective of the present investigation is to study the incursion of the Autonomous Juarez University of Tabasco (UJAT) based on the process of internationalization. This is done by analyzing the statistical information of the institutional program of the mobility of undergraduate students in period 2012-2016. The research approach is qualitative based on the documental analysis of the information contained in the Institutional Development Plans (IDP) 2012-2016 and 2016-2020, as well as in the annual reports of activities included in the study period. Statistics show that each year, the number of students increased with an increase of $69.6 \%$ when comparing the first year of the study with the year 2016. In order to determine the results of this study in absolute terms, the total enrollment of the UJAT in undergraduate degree in the years 2012 and 2016 was compared with the number of participating students, resulting in $0.17 \%$ and $0.53 \%$, respectively.
\end{abstract}

Keywords: international student mobility, public university, higher education 


\section{Resumen}

La internacionalización en educación superior como proceso complejo derivado de la nueva dinámica social, cultural y económica que se gesta en los contextos nacional e internacional, se concibe como una estrategia para garantizar a la sociedad un profesional competente y con mejores oportunidades laborales, a partir de la pertinencia social del currículo y el desarrollo de programas institucionales que así lo favorezcan. Para las Instituciones de Educación Superior (IES) incursionar en este proceso dinámico implica, según Knight (1994) la integración de las dimensiones internacional e intercultural en las funciones de enseñanza, investigación y servicios de la institución. En este sentido, el objetivo de la presente investigación es estudiar la incursión de la Universidad Juárez Autónoma de Tabasco (UJAT) en los procesos de internacionalización, mediante el análisis de la información estadística del programa institucional de la movilidad de los estudiantes de licenciatura en periodo 2012-2016. El enfoque de la investigación es cualitativo sustentado en el análisis documental de la información contenida en los Planes de Desarrollo Institucional (PDI) 20122016 y 2016-2020, así como en los informes anuales de actividades comprendidos en el periodo de estudio. Las estadísticas muestran que cada año se ha incrementado el número de alumnos; determinándose un incremento de $69.6 \%$ al comparar el primer año de estudio con el año 2016. A fin de determinar en términos absolutos este resultados, se compararon la matrícula total de la UJAT en licenciatura en los años 2012 y 2016 con el número de estudiantes participantes, resultando un $0.17 \%$ y $0.53 \%$, respectivamente.

Palabras Claves: movilidad estudiantil internacional, universidad pública, educación superior

\section{Introducción}

La Comisión Internacional de Educación creada por la Organización de las Naciones Unidas para la Educación, La Ciencia y la Cultura (UNESCO), plantea la necesidad de formar una cultura ciudadana que permita a los individuos participar en el desarrollo de la sociedad así como una educación fundamentada en conocimientos, valores, actitudes y aptitudes favorables para la construcción de una cultura democrática. Por lo que la educación es concebida como la única posibilidad de luchar contra la exclusión, la marginación y el desarrollo de personas y sociedades. En este contexto la calidad educativa, significa un factor de desarrollo y un medio fundamental para obtener mejores niveles de vida. De ahí la trascendente responsabilidad social y compromiso de las Instituciones de Educación Superior (IES), de formar profesionales competentes que logren su inserción en el mercado 
laboral de manera exitosa, aceptando el desafío de involucrarse y comprometerse con el desarrollo social, conscientes desde su rol como estudiantes de la importancia del análisis y reflexión dentro y fuera del aula del deber que tienen para coadyuvar a transformar su entorno y de ser sensibles a los problemas de los demás, y a proponer soluciones, que permitan la inclusión social de los más vulnerables.

Uno de los mecanismos que las IES para lograr el nivel de formación, involucramiento y compromiso social antes descrito de los profesionales, es precisamente la internacionalización de la educación superior, convirtiendo a la movilidad estudiantil, en una estrategia cuya finalidad consiste en ampliar los esquemas de formación profesional, logrando experiencias de aprendizaje y de vivencia en diversos contextos geográficos.

\section{Objetivo De La Investigación}

Analizar la incursión de la Universidad Juárez Autónoma de Tabasco (UJAT) en los procesos de internacionalización a partir de la información estadística de la participación de los estudiantes de licenciatura en el programa institucional de movilidad en el periodo 2012-2016.

\section{La Internacionalización En Educación Superior}

Hablar de internacionalización de la educación superior requiere de una revisión de las investigaciones realizadas para explicar esta forma de mejorar los procesos educativos, de investigación, difusión y vinculación de las IES, que favorezca la determinación de las dimensiones que han de precisar su significado así como sus implicaciones.

Un primer acercamiento al concepto de internacionalización de la educación superior, denota la complejidad del mismo, para Altbach (2004) la internacionalización incluye las políticas y programas específicos emprendidos por gobiernos, sistemas académicos, instituciones, y aun departamentos individuales, para bregar con la globalización o explotarla favorablemente. Van der Wende (1997) propuso que se considere la internacionalización como cualquier esfuerzo sistemático y sostenido dirigido a promover la respuesta de la educación superior a los requerimientos y desafíos relacionados con la globalización de las sociedades, la economía y el mercado de trabajo.

Ambas explicaciones del significado de la internacionalización de la educación superior, traslucen que es indispensable desarrollar mecanismos de respuesta a las múltiples necesidades sociales, desde los diferentes ámbitos funcionales de las Instituciones de Educación Superior. Al respecto, para Knight (1994) que la internacionalización de la educación, facilita la integración de la dimensión intercultural en la enseñanza y la investigación 
para colocarla tanto al servicio de la propia institución como de la sociedad a la cual sirve.

Por ello entonces, es que se ha afirmado que la internacionalización de la educación superior, es utilizada como estrategia por los países para responder a las repercusiones de la globalización aprovechando también sus beneficios de interculturalidad, sin perder la autenticidad de su propia cultura.

De esta forma coincidimos con lo señalado por Knight (2005), internacionalizar en educación lejos de poner en riesgo las identidades y culturas nacionales es reforzar la propia. Por lo que:

“...para que las condiciones de la educación superior internacional puedan hacerse realidad, los modelos educativos deberán considerar el contexto social, económico y político actual, así como las tendencias científicas, tecnológicas y culturales, que permitan diseñar escenarios y ambientes sustentables de educación, aprendizaje y retroalimentación permanente.” (Mapén, Rosas, Rodríguez \& Méndez, 2015, p. 151)

La finalidad entonces de la internacionalización de la educación superior, es generar nuevas dinámicas al interior de las instituciones, dentro de las cuales se incluyen, la pertinencia social del currículum impulsando de manera explícita en los planes y programas de estudios el desarrollo y fortalecimiento de competencias profesionales de nivel internacional.

México tiene razones importantes para impulsar la internacionalización de la educación superior, es por ello que a lo largo de los periodos se ha visto el crecimiento y la implementación de la movilidad estudiantil dentro de este nivel educativo, estrategia educativa que recientemente ha cobrado mayor impulso en la IES mexicanas, en tanto esta que en países desarrollados, esta actividad de intercambio se considera una parte fundamental y activa de la formación.

Como se ha podido observar, la movilidad académica es una de las estrategias que va conformando modelos de internacionalización, los cuales obedecen a razones económicas, culturales y sociales. En este sentido, Haug y Vilalta (2011) señalan tres modelos de internacionalización cuyas diferencias se encuentran en los procesos que se ejecutan en cada campus universitario. Tal y como se muestran en la siguiente tabla: 
Tabla 1. Modelos de Internacionalización

\begin{tabular}{|c|c|c|}
\hline $\begin{array}{c}\text { MODELO } \\
\text { TRADICIONAL }\end{array}$ & MODELO OFENSIVO & MODELO EMERGENTE \\
\hline $\begin{array}{ll}\text { Internacionalización } & \text { de } \\
\text { saberes y competencias. } & \end{array}$ & $\begin{array}{l}\text { Internacionalización basada } \\
\text { en la proximidad con los } \\
\text { medios profesionales y } \\
\text { empresariales. }\end{array}$ & $\begin{array}{l}\text { Internacionalización basada } \\
\text { en una transformación } \\
\text { general y progresiva }\end{array}$ \\
\hline $\begin{array}{l}\text { Basado en un elevado nivel } \\
\text { académico del profesorado. }\end{array}$ & $\begin{array}{l}\text { Alto nivel de exigencia } \\
\text { académica avalada por una } \\
\text { acreditación internacional. }\end{array}$ & \begin{tabular}{lcc} 
Programas & \multicolumn{2}{c}{ compartidos, } \\
institucionalización & de la \\
movilidad y & dobles \\
titulaciones. & & \\
\end{tabular} \\
\hline $\begin{array}{lll}\text { Impacto de una larga y } \\
\text { prestigiosa } & & \text { tradición } \\
\text { universitaria } & & \end{array}$ & $\begin{array}{l}\text { Profesores con presencia } \\
\text { internacional }\end{array}$ & $\begin{array}{l}\text { Mixto: } \\
\text { particularidades de amblican } \\
\text { modelos tradicional y } \\
\text { ofensivo. }\end{array}$ \\
\hline $\begin{array}{l}\text { Apuesta por la exigencia de } \\
\text { la excelencia. }\end{array}$ & $\begin{array}{l}\text { Reclutamiento de profesores } \\
\text { y estudiantes en cualquier } \\
\text { parte del mundo. }\end{array}$ & $\begin{array}{lr}\text { Mixto: } & \text { aplican } \\
\text { particularidades de ambos } \\
\text { modelos tradicional y } \\
\text { ofensivo. }\end{array}$ \\
\hline $\begin{array}{lll}\text { Creación de } & \text { redes } \\
\text { universitarias } & & \end{array}$ & $\begin{array}{lrr}\text { Fuertes } & \text { relaciones con } & \text { el } \\
\text { mundo } & \text { económico } & \text { y } \\
\text { orientación } & \text { profesional } & \text { de } \\
\text { los estudios } & & \end{array}$ & $\begin{array}{l}\text { Mixto: } \\
\text { particularidades de amblican } \\
\text { modelos tradicional y } \\
\text { ofensivo. }\end{array}$ \\
\hline $\begin{array}{l}\text { Frontera porosa entre el } \\
\text { mundo académico y el } \\
\text { profesional }\end{array}$ & $\begin{array}{l}\text { Sólidas políticas de } \\
\text { marketing y comunicación }\end{array}$ & $\begin{array}{l}\text { Creación de oficinas de } \\
\text { relaciones internacionales }\end{array}$ \\
\hline $\begin{array}{l}\text { Círculo cerrado de } \\
\text { profesores } \\
\text { comunidades científicas }\end{array}$ & $\begin{array}{l}\text { Estrechas relaciones con los } \\
\text { líderes políticos e } \\
\text { institucionales }\end{array}$ & $\begin{array}{l}\text { Mixto: } \\
\text { particularidades de ambos } \\
\text { modelos tradicional y } \\
\text { ofensivo. }\end{array}$ \\
\hline Lazos asociativos con pares. & $\begin{array}{lrr}\text { Política } & \text { formal } & \text { de } \\
\text { más quanzas } \\
\text { partenariado }^{23} & \text { numerosos } \\
\text { consistentes. } & \text { poco } \\
\end{array}$ & $\begin{array}{ll}\text { Alianzas } & \text { estratégicas } \\
\text { puntuales. } & \end{array}$ \\
\hline Campus “tradicional”. & $\begin{array}{l}\text { Campus “internacional” } \\
\text { Deslocalización. }\end{array}$ & Campus virtuales. \\
\hline $\begin{array}{l}\text { Riesgo de desvincularse de } \\
\text { las expectativas de los } \\
\text { estudiantes }\end{array}$ & $\begin{array}{l}\text { Costosa, arriesgada y alejada } \\
\text { de la tradición universitaria }\end{array}$ & $\begin{array}{l}\text { Adaptación de las } \\
\text { estructuras de gobierno, de } \\
\text { financiación y de ordenación } \\
\text { académica }\end{array}$ \\
\hline
\end{tabular}

Fuente: Recuperado de Haug G. y Vilalta (2011). Documento de Trabajo/Working paper 2. Studia XXI. Fundación Europea Sociedad y Educación.

\section{Como se observa, el modelo tradicional se conforma por acciones que se}

${ }^{23}$ Según el Programa de Naciones Unidas para el Desarrollo (PNUD), el partenariado es una forma de entender el desarrollo desde la participación, a través del diálogo y negociación entre los diversos actores que establecen un programa de acciones conjuntas. De manera que los beneficiarios se conviertan en ejecutores de la acción de desarrollo. 
llevan a cabo en un plano estrictamente académico, enfatizando en la movilidad e intercambio de estudiantes y profesores. El modelo ofensivo destaca el proceso en función de la vinculación que se establezca con los medios profesionales y empresariales, haciendo del diálogo y la negociación los mecanismos que han de atraer a los mejores profesores y estudiantes, considerando incluso establecer campus en el extranjero.

El modelo emergente de internacionalización según Mapén et al. (2015) responde a formas híbridas de los modelos anteriores en donde sus acciones comprenden doble titulación en los programas educativos de sus ofertas educativas así como en las diversas modalidades -presenciales y virtuales-, creación de oficinas de relaciones internacionales e incluso campus virtuales.

Este breve análisis de los modelos nos obliga a reflexionar a las causas que impulsan a las IES a desarrollar estrategias de internacionalización y las encontramos en la propia institucionalidad de las mismas, ya que son concebidas como la culminación de una formación profesional que debe ser pertinente garantizando con éxito la inserción laboral de los individuos a través de las competencias desarrolladas.

La movilidad internacional de estudiantes universitarios se inscribe en tendencias culturales de mayor alcance para lograr la difusión de una cultura juvenil de la movilidad, motivada menos por los tradicionales factores económicos de la migración como son el empleo, y más por factores de educación, tiempo libre y adquisición de experiencia; constituye por tanto, un fenómeno de creciente importancia, cuyo impacto es notorio en los jóvenes universitarios.

La internacionalización tiene como apoyo herramientas como la vinculación y movilidad dentro de los estudiantes lo cual es un proceso de cambio diseñado a medida para satisfacer las necesidades y los intereses de cada entidad de educación superior. En consecuencia, no existe un modelo de internacionalización que se adapte a todos por igual.

La movilidad universitaria ha tenido grandes resultados para el desarrollo de programas que buscan crear un modelo para ampliar el conocimiento y enfocarlo de manera global a los alumnos, pues la internacionalización ha tenido gran impacto en el mundo de la educación superior.

Los recientes estudios nacionales e internacionales sobre las prioridades y las bases lógicas de la internacionalización universitaria demuestran que establecer un perfil internacional es más importante que cumplir los estándares internacionales de excelencia.

El desarrollo de capacidades en los universitarios a través de proyectos de cooperación internacional está siendo remplazado por iniciativas encaminadas a reforzar el reconocimiento mundial.

El impacto del proceso de movilidad de la educación superior puede analizarse desde una perspectiva de los beneficios y experiencias que se 
obtienen mediante este proceso, el cual permite cumplir estándares de éxito en los egresados universitarios que realizaron movilidad estudiantil y los beneficios que obtuvieron al incursar en el ámbito profesional y laboral.

En estudios realizados se destacan los beneficios en el profesional en el desarrollo de habilidades adquiridas mediante el proceso, así como en la mejor de las relaciones sociales al momento de incursar en la competencia laboral.

Por lo que la incursión de las universidades en la internacionalización es todo un proceso de evolución, retos y desafíos cuya complejidad adopta formas distintas dependiendo de las particularidades de las IES, en donde el tema de financiamiento, ocupa un lugar preponderante.

\section{La UJAT y su incursión a los procesos de internacionalización.}

La UJAT es una institución pública de educación superior conformada por doce divisiones académicas distribuidas en diferentes municipios del estado de Tabasco, México: Ciencias Agropecuarias (DACA), Ciencias Económico-Administrativas (DACEA), Educación y Artes (DAEA), Ciencias Sociales y Humanidades (DACS y H), Ingeniería y Arquitectura (DAIA), Informática y Sistemas (DAIS), Ciencias de la Salud (DACS), Ciencias Biológicas (DACBIOL), Ciencias Básicas (DACB), Multidisciplinaria de Comalcalco (DAMC), Multidisciplinaria de Jalpa de Méndez (DAMJ) y Multidisciplinaria de los Ríos (DAMR) en los que se atiende a 29, 866 estudiantes en licenciatura y 1061 en estudios de posgrado (UJAT, 2016a). Su filosofía se define, principalmente a través de su misión y visión, la primera se enfoca a:

"contribuir de manera significativa a la transformación de la sociedad y al desarrollo del país, con particular interés en el Estado de Tabasco, a través de la formación sólida e integral de profesionales capaces de adquirir, generar, difundir y aplicar el conocimiento científico, tecnológico y humanístico, con ética y responsabilidad para ser mejores individuos y ciudadanos” (UJAT,2016a, p. 41).

En cuanto a su visión al 2020 se concibe como "una institución con presencia nacional e internacional, socialmente responsable e incluyente, que contribuye al desarrollo sostenible de Tabasco y México, mediante programas educativos de calidad que forman profesionales éticos y competitivos en el ámbito global. Se distingue por sus relevantes aportes en la transferencia de ciencia y tecnología, el respeto y promoción de la cultura y la vinculación efectiva con su entorno, en el marco de una gestión moderna que promueve los derechos humanos, la salud integral, la transparencia y rendición de cuentas.” (UJAT, 2016a, p. 41)

El logro de ambas se define a través de estrategias y acciones que compromete en el Plan de Desarrollo Institucional (PDI) (2016-2020) para 
garantizar a la sociedad la prestación de un servicio educativo de calidad, experiencias exitosas, esquemas y tendencias para generar y aplicar el conocimiento e innovaciones tecnológicas, la colaboración en redes académicas y de investigación, impulso al diálogo intercultural, la inclusión social con equidad y articularse con los sectores sociales y productivos para coadyuvar al desarrollo de la región y el país. Para ello ha adoptado a la internacionalización y la responsabilidad social como ejes transversales que refuercen todas las funciones institucionales docencia, investigación, extensión y difusión de la cultura, vinculación para el desarrollo gestión moderna- y la haga presente en escenarios extranjeros como formadores de ciudadanos del mundo que comparten experiencias y conocimientos, en ámbitos de diversidad, con el propósito de ofrecer alternativas innovadoras ante las problemáticas globales.

Para ello se han definido las siguientes estrategias (UJAT, 2016a) que pretender dar respuesta al reto planteado en el PDI (2012-2016), enfocado a la transcendencia en el ámbito mundial:

- Impulsar la creación de programas de movilidad y formación del personal administrativo con instituciones nacionales $\mathrm{e}$ internacionales.

- Fomentar la organización de eventos académicos institucionales que incidan en la consolidación de la cooperación académica nacional e internacional.

- $\quad$ Apoyar la integración de la Universidad a redes académicas y de investigación internacionales.

- Establecer alianzas con instituciones u organismos internacionales que sean afines a las necesidades y retos de la Universidad.

Institucionalizar la dimensión internacional como eje transversal de las funciones universitarias, consolidando el marco normativo y la estructura organizacional correspondientes, así como dando seguimiento a la consecución de los indicadores establecidos para este rubro.

- $\quad$ Propiciar la homologación de los programas y procedimientos institucionales adoptando normas internacionales de auditoría en el marco de las nuevas disposiciones fiscales para las universidades públicas.

Como se observa, la movilidad estudiantil internacional es considerada una estrategia institucional que pretende fortalecer la formación profesional de estudiantes a través del fomento de competencias sociales y laborales que permitan una incursión exitosa en el ámbito laboral. 
La participación en redes de colaboración académica ha favorecido la movilidad estudiantil, que de manera internacional se ha logrado a través de los siguientes convenios:

- Programa Académico de Movilidad Educativa: Unión de Universidades de América Latina y el Caribe: el intercambio estudiantil se establece entre las universidades adscritas; la universidad receptora otorga la beca de apoyo al estudiante durante su estancia y la UJAT realiza los pagos de boletos de avión viaje redondo.

- Consorcio para la Colaboración de la Educación Superior en América del Norte: los estudiantes pueden realizar sus estancias académicas en países como: Argentina, Brasil, Chile, Colombia, República Dominicana, Guatemala, Honduras, Islandia, Corea, Burkina Faso y España. En este caso la UJAT otorga la beca de apoyo que incluye el pago de los boletos de avión y viáticos.

- Programa de Intercambio BRAMEX-ANUIES: promueve el intercambio de estudiantes de nivel superior entre las instituciones miembros de la Asociación Nacional de Universidades e Instituciones de Educación Superior (ANUIES) y Grupo Coimbra de Universidades Brasileñas. Este programa establece como obligatorio el hecho de que las instituciones de educación superior (IES), públicas y privadas participantes, movilicen al menos dos estudiantes por semestre entre ambas naciones sin distinción del programa académico que cursen. La Universidad receptora otorga beca de apoyo al estudiante que incluye el pago de la cuota de inscripción, hospedaje, alimentación y la UJAT paga los boletos de avión, viaje redondo.

- Programa Mexfitec (México- Francia- Ingenieros-Tecnología): dirigido a estudiantes mexicanos de ingeniería que deseen enriquecer su formación profesional en una universidad francesa.

Estos convenios han favorecido que cada año se incremente el número de estudiantes que de manera voluntaria desean participar en el programa de movilidad internacional.

De esta manera, a través de la movilidad estudiantil la UJAT impulsa a sus estudiantes al logro de enfoques diversos respecto a las formas de desarrollo social, cultural, económicos y demás competencias que favorezcan la mejora de su formación y desempeño profesional. De tal manera que la movilidad estudiantil es concebida como una fortaleza para ampliar las perspectivas de formación mediante la exposición a contextos académicos y culturales diferentes.

\section{Metodología}

La investigación es de tipo cualitativo (Hernández S., Fernández C. \& Baptista L., 2014) sustentado en la técnica de análisis documental; 
determinándose como población de estudio, los estudiantes que participaron en el programa de movilidad internacional de 2012 a 2016.

Para conocer los resultados alcanzados en la materia de movilidad académica internacional de estudiantes, se analizaron los PDI (2012-2016) y (2016-2020) a fin de identificar las estrategias planteadas para el logro de la internacionalización de la UJAT, así como los informes anuales del rector de la UJAT durante el periodo de estudio, de tal manera que fuera posible corroborar a través de datos estadísticos históricos la participación de los estudiantes en el programa de movilidad internacional así como los convenios de colaboración internacional que la universidad ha establecido con universidades del extranjero para posibilitar el intercambio de estudiantes.

Así también en forma paralela se obtuvo información del departamento de movilidad estudiantil, la cual permitió identificar la participación de los estudiantes en las diferentes divisiones académicas que conforman la UJAT.

\section{Resultados}

Con base a lo anterior se determinó el número de estudiantes que participaron en el programa de movilidad internacional de 2012 a 2016. Como se observa en la tabla 2, cada año se ha incrementado el número de alumnos; determinándose un incremento de 69.6\% al comparar el primer año de estudio con el año 2016. A fin de determinar en términos absolutos este resultados, se compararon la matrícula total de la UJAT en licenciatura en los años 2012 y 2016 con el número de estudiantes participantes, resultando un $0.17 \%$ y $0.53 \%$, respectivamente.

Tabla 2. Número de estudiantes que participaron en movilidad internacional en el periodo de estudio 2012-2016

\begin{tabular}{|c|c|c|}
\hline AÑO & $\begin{array}{c}\text { NÚMERO DE } \\
\text { ESTUDIANTES }\end{array}$ & PAÍSES \\
\hline 2012 & 48 & $\begin{array}{c}\text { Canadá, Estados Unidos de Norteamérica, } \\
\text { Brasil, Colombia, Chile, Argentina, } \\
\text { España, Francia e Italia. }\end{array}$ \\
\hline 2013 & 96 & \\
\hline 2014 & 138 & \\
\hline 2015 & 150 & \\
\hline 2016 & 158 & \\
\cline { 1 - 2 } TOTAL & $\mathbf{5 9 0}$ &
\end{tabular}

Fuente: Elaboración propia con información proporcionada por el Departamento de Movilidad Estudiantil de la UJAT.

En las tablas subsecuentes se presenta a detalle la participación de los estudiantes considerando la división académica en la que estaban o están 
inscritos, ya que actualmente algunos ya egresaron. Es importante destacar que en cada uno de los años de estudio los estudiantes de DAEA han demostrado mayor interés en realizar movilidad internacional, ya el número mayor de participantes se concentra en esta división, en 2012 representaron el 50\% del total de participantes, en 2013 el 61.5\%, en 2014 el 34\%, en 2015 el 22\% y en 2016 el 26.2\%. De manera constante los estudiantes de DACEA, ocupan el segundo lugar en participar en el programa de movilidad internacional.

Otro resultado de la investigación a destacar es que en ninguno de los años de estudio han participado estudiantes de la DACB, en la que se imparten las siguientes licenciaturas: Actuaría, Ciencias Computacionales, Física, Matemáticas e Ingeniería en Geofísica. De igual manera aún no hay participantes de la DAMJ en donde se ofertan las licenciaturas: Enfermería, Ingeniería Petroquímica, ingeniería en Nanotecnología y la Licenciatura en Genómica; sin embargo, es necesario considerar que esta división académica es la de más reciente creación -Abril de 2014-. En ambos casos se consideran necesarias acciones que motiven a los estudiantes a realizar estancias académicas en el extranjero a fin de fortalecer sus competencias profesionales.

Tabla 3. Alumnos que participaron en Movilidad Internacional en el año 2012 de cada División Académica.

\begin{tabular}{|c|c|c|c|c|c|c|c|}
\hline PAÍSES & $\begin{array}{c}\text { DACE } \\
\text { A }\end{array}$ & DAEA & $\begin{array}{c}\text { DACBI } \\
\text { OL }\end{array}$ & DAIS & $\begin{array}{c}\text { DACSy } \\
\text { H }\end{array}$ & DAIA & TOTAL \\
\hline $\begin{array}{c}\text { España } \\
\text { Francia }\end{array}$ & 12 & 24 & 4 & 2 & 2 & 4 & $\mathbf{4 8}$ \\
\hline
\end{tabular}

\begin{tabular}{|c|c|c|c|c|c|c|c|c|c|c|}
\hline PAÍSES & $\begin{array}{c}\text { DA } \\
\text { CE } \\
\text { A }\end{array}$ & $\begin{array}{c}\text { DAI } \\
\text { A }\end{array}$ & $\begin{array}{c}\text { DAC } \\
\text { A }\end{array}$ & DAEA & $\begin{array}{c}\text { DACBI } \\
\text { OL }\end{array}$ & $\begin{array}{c}\text { DACS } \\
\text { yH }\end{array}$ & $\begin{array}{c}\text { DAI } \\
\mathrm{S}\end{array}$ & $\begin{array}{c}\text { DAM } \\
\text { C }\end{array}$ & $\begin{array}{c}\text { DAC } \\
\text { S }\end{array}$ & $\begin{array}{c}\text { TOTA } \\
\text { L }\end{array}$ \\
\hline $\begin{array}{c}\text { Argentina } \\
\text { Brasil } \\
\text { Canadá } \\
\text { Chile } \\
\text { Colombia } \\
\text { España } \\
\text { Estados } \\
\text { Unidos de } \\
\text { Nortea- } \\
\text { mérica } \\
\text { Francia }\end{array}$ & 10 & 2 & 2 & 59 & 3 & 6 & 3 & 2 & 9 & 96 \\
\hline
\end{tabular}

Fuente: Elaboración propia con base en la información proporcionada por el Departamento de Movilidad Estudiantil de la UJAT y el Informe Anual de Actividades 2012 (UJAT, 2013). 
Tabla 4. Alumnos que participaron en Movilidad Internacional en el año 2013 de cada División Académica

\begin{tabular}{|c|c|c|c|c|c|c|c|c|c|c|c|}
\hline PAÍSES & $\begin{array}{c}\text { DAC } \\
\text { EA }\end{array}$ & $\begin{array}{c}\text { DAI } \\
\text { A }\end{array}$ & $\begin{array}{c}\text { DAC } \\
\text { A }\end{array}$ & $\begin{array}{l}\text { DA } \\
\text { EA }\end{array}$ & $\begin{array}{c}\text { DA } \\
\text { C- } \\
\text { BIO } \\
\text { L }\end{array}$ & $\begin{array}{l}\text { DA } \\
\text { CS } \\
\text { yH }\end{array}$ & DAIS & $\begin{array}{c}\text { DAM } \\
\text { C }\end{array}$ & $\begin{array}{l}\text { DA } \\
\text { CS }\end{array}$ & $\begin{array}{c}\text { DAM } \\
\text { R }\end{array}$ & $\begin{array}{c}\text { TOTA } \\
\text { L }\end{array}$ \\
\hline $\begin{array}{l}\text { Argentina } \\
\text { Brasil } \\
\text { Canadá } \\
\text { Chile } \\
\text { Colombia } \\
\text { España } \\
\text { Estados } \\
\text { Unidos de } \\
\text { Norteaméri } \\
\text { ca Francia }\end{array}$ & 28 & 20 & 2 & 47 & 10 & 9 & 3 & 8 & 9 & 2 & 138 \\
\hline
\end{tabular}

Fuente: Elaboración propia con base en la información proporcionada por el Departamento de Movilidad Estudiantil de la UJAT y el Informe Anual de Actividades 2013 (UJAT, 2014).

Tabla 5. Alumnos que participaron en Movilidad Internacional en el año 2014 de cada División Académica

\begin{tabular}{|c|c|c|c|c|c|c|c|}
\hline PAÍSES & $\begin{array}{c}\text { DACE } \\
\text { A }\end{array}$ & DAEA & $\begin{array}{c}\text { DACBI } \\
\text { OL }\end{array}$ & DAIS & $\begin{array}{c}\text { DACSy } \\
\text { H }\end{array}$ & DAIA & TOTAL \\
\hline $\begin{array}{c}\text { España } \\
\text { Francia }\end{array}$ & 12 & 24 & 4 & 2 & 2 & 4 & $\mathbf{4 8}$ \\
\hline
\end{tabular}

Fuente: Elaboración propia con base en la información proporcionada por el Departamento de Movilidad Estudiantil de la UJAT y el Informe Anual de Actividades 2014 (UJAT, 2015).

Tabla 6. Alumnos que participaron en Movilidad Internacional en el año 2015 de cada División Académica.

\begin{tabular}{|c|c|c|c|c|c|c|c|c|c|c|c|}
\hline PAÍSES & $\begin{array}{c}\mathrm{D} \\
\mathrm{A} \\
\mathrm{CE} \\
\mathrm{A}\end{array}$ & $\begin{array}{l}\text { DA } \\
\text { IA }\end{array}$ & $\begin{array}{c}\text { DAC } \\
\text { A }\end{array}$ & $\begin{array}{c}\text { DAE } \\
\text { A }\end{array}$ & $\begin{array}{c}\text { DACB } \\
\text { IOL }\end{array}$ & $\begin{array}{c}\text { DAC } \\
\text { SyH }\end{array}$ & $\begin{array}{c}\text { DAI } \\
\mathrm{S}\end{array}$ & $\begin{array}{l}\text { DA } \\
\text { MC }\end{array}$ & DACS & DAMR & Total \\
\hline $\begin{array}{l}\text { Argentina } \\
\text { Brasil } \\
\text { Canadá } \\
\text { Chile } \\
\text { Colombia } \\
\text { España } \\
\text { Estados } \\
\text { Unidos de } \\
\text { Nortea- } \\
\text { mérica } \\
\text { Francia }\end{array}$ & 40 & 20 & 4 & 44 & 7 & 18 & 1 & 10 & 22 & 2 & 168 \\
\hline
\end{tabular}

Fuente: Elaboración propia con base en la información proporcionada por el Departamento de Movilidad Estudiantil de la UJAT y el Informe Anual de Actividades 2015 (UJAT, 2016b). 
Table 7. Alumnos que participaron en Movilidad Internacional en el año 2016 de cada División Académica

\begin{tabular}{|c|c|c|c|c|c|c|c|c|c|c|c|}
\hline PAÍSES & $\begin{array}{l}\mathbf{D} \\
\mathbf{A} \\
\mathbf{C} \\
\mathbf{E} \\
\mathbf{A}\end{array}$ & $\begin{array}{c}\text { D } \\
\text { AI } \\
\text { A }\end{array}$ & $\begin{array}{l}\text { DA } \\
\text { CA }\end{array}$ & $\begin{array}{l}\text { DA } \\
\text { EA }\end{array}$ & $\begin{array}{c}\text { D } \\
\text { A } \\
\text { CB } \\
\text { IO } \\
\text { L }\end{array}$ & $\begin{array}{c}\text { DA } \\
\text { CSy } \\
\text { H }\end{array}$ & $\begin{array}{c}\text { DAI } \\
\text { S }\end{array}$ & $\begin{array}{l}\text { DA } \\
\text { MC }\end{array}$ & $\begin{array}{l}\text { DA } \\
\text { CS }\end{array}$ & $\begin{array}{l}\text { DS } \\
\text { MR }\end{array}$ & $\begin{array}{c}\text { TOT } \\
\text { AL }\end{array}$ \\
\hline $\begin{array}{l}\text { Argentina } \\
\text { Brasil } \\
\text { Canada } \\
\text { Chile } \\
\text { Colombia } \\
\text { España } \\
\text { Estados } \\
\text { Unidos de } \\
\text { Nortea- } \\
\text { mérica } \\
\text { Francia }\end{array}$ & 32 & 18 & 5 & 33 & 16 & 20 & 2 & 10 & 12 & 2 & 150 \\
\hline
\end{tabular}

Fuente: Elaboración propia con base en la información proporcionada por el Departamento de Movilidad Estudiantil de la UJAT y el Informe Anual de Actividades 2016 (UJAT, 2017).

\section{Conclusión}

Los cambios acelerados que se suscitan a medida que avanzamos en el siglo XXI en materia económica, social, cultura y tecnológica hacen que la internacionalización en educación superior sea cada vez más importante pero también más compleja. Las particularidades de cada IES representan un conjunto de oportunidades y/o desafíos que enfrentar, por lo que no existe una ruta de acción estandarizada que posibilite la internacionalización ni un conjunto de indicadores que permitan establecer parámetros al respecto.

Sin embargo existe cierto consenso al considera la movilidad estudiantil como estrategia formativa de sus estudiantes y uno de los primeros peldaños para adentrarse en la dimensión internacional en educación superior. En este sentido la UJAT ha desarrollado una serie de acciones en las que se destacan la firma de convenios y participación en redes de intercambio considerando países con idioma diferente al español a partir de 2012. Mecanismos de respuesta que coinciden con el modelo tradicional de internacionalización y que nos permiten afirmar que la incursión de la universidad en los procesos de internacionalización es incipiente.

\section{References:}

1. Altbach, P.G. (2004). Globalization and the university: Myths and realities in an unequal world. In National Education Association (Ed.), The NEA 2005 almanac of higher education (pp. 63-74). 
Washington, DC: National Education Association. Recuperado de: http://199.223.128.150/assets/img/PubAlmanac/ALM_05_06.pdf.

2. Haug G. \& Vilalta (2011). “La internacionalización de las Universidades, una estrategia necesaria. Una reflexión sobre la vigencia de modelos académicos, económicos y culturales en la gestión de la internacionalización universitaria”. Documento de Trabajo/Working paper 2. Studia XXI. Fundación Europea Sociedad y Educación.

3. Hernández Sampieri, R., Fernández Collado, C. \& Baptista Lucio, M. ed., (2014). Metodología de la Investigación, 6th Ed. México, D.F. Mac Graw-Hill Interamericana Editores S.A. de C.V.

4. Knight, J. (1994). Internationalization: Elements and checkpoints CBIE Research, No. 7, Canadian Bureau for International Education, 3-4. Recuperado de: https://archive.org/stream/ERIC_ED549823\#page/n13/mode/2up.

5. __ (2005). Un modelo de internacionalización: respuesta a nuevas realidades y retos en Educación Superior en América Latina: la dimensión internacional. Banco Mundial.

6. Mapén, F. F., Rosas, C. J., Rodríguez, G. C. y Méndez, M. W. (2015). Análisis organizacional de la internacionalización de las universidades públicas estatales mediante la movilidad y el intercambio académicos en El Análisis Organizacional en México y América Latina: retos y perspectivas a 20 años de estudios. Primera edición. (pp. 145-170). Ed. Hess.

7. Universidad Juárez Autónoma de Tabasco [UJAT] (2012). Plan de Desarrollo Institucional 2012-2016. Villahermosa, Tabasco. Autor. Recuperado de http://www.ujat.mx/rectoria/23502

8. _ (2013). 1er. Informe de Actividades (2012). Dr. Jesús Manuel Piña Gutiérrez. Rector. Villahermosa, Tabasco. Autor. Recuperado de

http://www.archivos.ujat.mx/2013/rectoria/informe_actividades/1er_i nforme_2012.pdf

9. (2014). 2do. Informe de Actividades 2013. Dr. Jesús Manuel Piña Gutiérrez. Rector. Villahermosa, Tabasco. Autor. Recuperado de

http://www.archivos.ujat.mx/2014/Rectoria/2do_informe/SEGUNDO \%20INFORME\%20UJAT\%202013.pdf

10. (2015). 3er. Informe de Actividades 2014. Dr. Jesús Manuel Piña Gutiérrez. Rector. Villahermosa, Tabasco. Autor. Recuperado de

http://www.archivos.ujat.mx/2015/rectoria/informe/TERCER\%20IN FORME\%202014.pdf 
11. (2016a). El Plan de Desarrollo a Institucional 2016 - 2020. Villahermosa, Tabasco. Autor. Recuperado de http://www.ujat.mx/rectoria/7119

12. ___ (2016b). 4to. Informe de Actividades 2015. Dr. Jesús Manuel Piña Gutiérrez. Rector. Villahermosa, Tabasco. Autor. Recuperado de

http://www.archivos.ujat.mx/2016/rectoria/4to_informe/COMPLETO /IV\%20INFORME\%202015\%20COMPLETO\%20FINAL.pdf

13. (2017). 1er. Informe de Actividades 2016. Dr. Jesús Manuel Piña Gutiérrez. Rector. Villahermosa, Tabasco. Autor. Recuperado de

http://www.archivos.ujat.mx/2017/Rectoria/1er\%20informe\%202017 /1ER\%20INFORME\%20DE\%20ACTIVIDADES_COMPLETO_20 17.pdf

14. Van der Wende, M. (1997). Internationalizing the Curriculum in Dutch Higher Education: an international comparative perspective, Nuffic (the Netherlands Organisation for International Cooperation in Higher Education), Academic Cooperation Association (ACA) in Brussels, 8. DOI:https://doi.org/10.1177/102831539700100204 